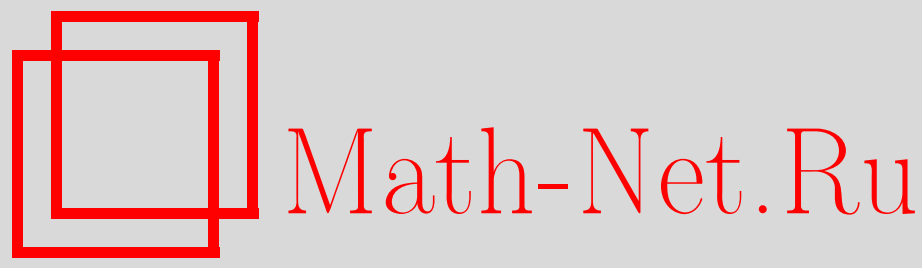

А. Н. Стародубцев, Фазовое пространство гравитирующей частицы и размерная редукция на планковских масштабах, ТМФ, 2015, том 185, номер 1, 192-198

DOI: https://doi.org/10.4213/tmf8928

Использование Общероссийского математического портала Math-Net.Ru подразумевает, что вы прочитали и согласны с пользовательским соглашением http://www.mathnet.ru/rus/agreement

Параметры загрузки:

IP : 35.173 .219 .12

26 апреля 2023 г., 11:33:00

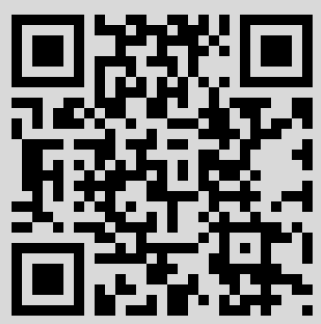




\title{
ФАЗОВОЕ ПРОСТРАНСТВО ГРАВИТИРУЮЩЕЙ ЧАСТИЦЫ И РАЗМЕРНАЯ РЕДУКЦИЯ НА ПЛАНКОВСКИХ МАСШТАБАХ
}

\begin{abstract}
Ряд походов к квантованию общей теории относительности указывает на то, что квантовая гравитация на очень малых расстояниях эффективно ведет себя как двумерная теория. Механизм этой редукции пока остается непонятым. Предпринята попытка объяснить его на основе изучения фазового пространства пробной частицы, взаимодействующей с гравитационным полем. Уравнения связей общей теории относительности позволяют выразить энергию-импульс частицы через некоторые инварианты кривизны, принимающие значения на групповом многообразии. Некоторые направления в полученном импульсном пространстве оказываются компактными, что ведет к "обратной редукции Калуцы-Клейна" на малых расстояниях.
\end{abstract}

Ключевые слова: квантовая гравитация, петли Вильсона, магнитный монополь, искривленное импульсное пространство.

DOI: $10.4213 / \operatorname{tmf} 8928$

\section{1. ВВЕДЕНИЕ}

Различные подходы к квантовой гравитации предсказывают, что на планковских масштабах пространство-время становится эффективно двумерным. Среди этих подходов упомянем "причинные динамические триангуляции" [1], "сценарий асимптотической безопасности" [2] и некоторые другие. Мы не описываем их здесь подробно и предлагаем работу [3] в качестве обзора. Эти подходы применялись к гравитации в пространствах размерности $2+1,3+1$ и более, и микроскопическая размерность пространства-времени всегда сводилась к двум.

Хотя свидетельства в пользу размерной редукции, полученные из нескольких независимых источников, выглядят убедительно, нам не хватает наглядного объяснения механизма этой редукции. Подходы к квантовой гравитации, основанные на универсальности и (или) численных методах, оставляют этот механизм неясным.

* Санкт-Петербургский государственный университет, Санкт-Петербург, Россия. E-mail: artemstarodubtsev@gmail.com 
В попытке объяснить исчезновение некоторых измерений примем самое наглядное определение размерности. Разместим в пространстве пробную частицу. Размерностью пространства будет число степеней свободы этой частицы.

Если гравитация и взаимодействующая с ней частица являются единственными ингредиентами теории, ка́к в принципе может произойти изменение размерности пространства? Внешнее гравитационное поле может компактифицировать некоторые из измерений пространства, в котором движется частица. Это хорошо известная редукция Калуцы-Клейна, которая происходит, однако, на больших масштабах. На малых масштабах внешнее гравитационное поле не имеет никакого влияния.

Единственная оставшаяся возможность - это учет гравитационного поля, создаваемого само́й пробной частицей. Обычно этим полем принято пренебрегать, что допустимо в большинстве практических ситуаций. Однако, как впервые было замечено Бронштейном в 1935 г. [4], когда мы производим измерение на планковском масштабе, мы не можем пренебрегать гравитационным полем, созданным этим измерением. Этот эффект обратной реакции налагает ограничение на масштаб, на котором измерение возможно в принципе. Главная трудность заключается в том, что вышеупомянутый эффект не виден в теории возмущений. Поэтому неудивительно, что все подходы к квантовой гравитации, перечисленные в работе [3], которые предсказывают размерную редукцию на малых масштабах, непертурбативны. Таким образом, естественно начать изучение этого эффекта в рамках точно решаемой теории.

В разделе 2 мы рассматриваем результаты для $(2+1)$-мерной гравитации, взаимодействующей с точечными частицами, полученные ранее рядом авторов [5], [6]. Теория точно решаема, и результат обратной реакции гравитационного поля на частицу заключается в том, что такая частица имеет кривое импульсное пространство. Кривизна импульсного пространства постоянна, ее радиус определяется планковской энергией, и одно из трех измерений в этом пространстве компактно.

В разделе 3 мы распространяем часть этих результатов на $(3+1)$-мерную гравитацию, взаимодействующую с единственной точечной частицей. Хотя полная теория не решаема точно, для наших целей достаточно рассмотреть одно частное решение, которое известно. Импульсное пространство частицы снова кривое. Снова это пространство постоянной кривизны, но его радиус кривизны теперь зависит от положения частицы в координатном пространстве, а именно от расстояния до начала отсчета. При фиксированном расстоянии до начала отсчета радиус кривизны импульсного пространства - это такой масштаб энергии, для которого это расстояние является радиусом Шварцшильда. Из четырех измерений в этом импульсном пространстве только два некомпактны.

\section{2. ИМПУЛЬСНОЕ ПРОСТРАНСТВО ЧАСТИЦЫ В $(2+1)$-МЕРНОЙ ГРАВИТАЦИИ}

$(2+1)$-мерная гравитация - это теория Черна-Саймонса для группы $I S O(2,1)$. Пусть $\langle\cdot\rangle$ - антидиагональная билинейная форма, которая перемешивает трансляционную и лоренцеву части алгебры $i s o(2,1)$. Ниже $A-I S O(2,1)$-связность, $K-$ фиксированный элемент алгебры $i s o(2,1)$, класс сопряженности которого определяет массу и спин частицы, $h$ - переменная, принимающая значения в группе $I S O(2,1)$, a $\gamma$ обозначает мировую линию частицы. Действие гравитации, взаимодействующей 
с частицей, имеет вид

$$
S=\kappa \int_{M}\left\langle A \wedge d A+\frac{2}{3} A \wedge A \wedge A\right\rangle+\int_{\gamma}\left\langle h^{-1} d_{A} h K\right\rangle,
$$

где $d_{A}$ - ковариантная внешняя производная со связностью $A$. Отсюда мы можем вывести уравнение связи

$$
C=\kappa F(A)-h K h^{-1} \delta^{2}\left(x-x_{\mathrm{p}}\right)=0 .
$$

Применяя неабелеву теорему Стокса [7] к последнему уравнению, можно связать внешний заряд $K$ с величиной петли Вильсона $g$ вокруг мировой линии частицы:

$$
h e^{K / \kappa} h^{-1}=g \text {. }
$$

Можно также получить эффективное действие для частицы, которое учитывает гравитационную обратную реакцию [6], [8]. Для этого решение уравнения связи

$$
A=\gamma(K d \phi+d) \gamma^{-1}, \quad \gamma\left(x_{0}\right)=\mathrm{Id}, \quad \gamma\left(x_{\mathrm{p}}\right)=h,
$$

подставляется в действие

$$
S=\kappa \int_{M}\langle A \dot{A}\rangle+\int_{\gamma}\left\langle h^{-1} \dot{h} K\right\rangle+\int_{\neg M}\left\langle A_{0} \dot{A}_{0}\right\rangle,
$$

где последний член - граничное действие, в котором $A_{0}-$ плоская связность, $\neg M-$ дополнение $M$ до всего пространства. Результат подстановки выглядит следующим образом:

$$
S=\kappa \int \operatorname{Tr}\left(x u^{-1} \dot{u}\right)
$$

где $u$ - лоренцева часть $g$, а $x$ - трансляционная часть $h$. Можно видеть, что $u$ играет роль импульса и является элементом группы $S O(2,1)$. Он удовлетворяет уравнению массовой поверхности

$$
\operatorname{Tr} u-\cos \frac{m}{\kappa}=0 .
$$

Многообразие группы $S O(2,1)$ имеет одно компактное направление, которое соответствует пространственным поворотам.

Конструкции, рассмотренные в этом разделе, были подробно изучены и обобщены в рамках так называемой “двойной специальной теории относительности” [9].

\section{3. РЕЗУЛЬТАТЫ ДЛЯ $(3+1)$-МЕРНОЙ ГРАВИТАЦИИ}

Что может нам помешать распространить вышеописанные результаты на $(3+1)$ мерную гравитацию? Прежде всего, $(3+1)$-мерная гравитация имеет бесконечное число степеней свободы - гравитационные волны. Однако, если мы рассмотрим ситуацию с единственной свободно движущейся частицей, гравитационные волны излучаться не будут. Другими словами, мы можем рассмотреть "минисуперпространственную" модель, которая получается замораживанием всех степеней свободы, кроме конечного их числа. Степенями свободы, оставшимися незамороженными, являются координаты частицы по отношению к некоторому выбранному началу отсчета. 
Вторая проблема заключается в том, что, в отличие от двумерной ситуации, точечная частица в трех пространственных измерениях не характеризуется топологически выделенной петлей Вильсона, которая содержала бы информацию об импульсе частицы. В действительности такая петля существует, однако, чтобы увидеть ее явно, нужно проделать некоторые манипуляции с решением. Наиболее естественно это можно сделать в рамках чисто калибровочной формулировки теории $(3+1)$ мерной гравитации. Это формулировка Макдауэлла-Мансури [10], которая во многом аналогична формулировке Черна-Саймонса для $(2+1)$-мерной гравитации, но применима только в случае ненулевой космологической постоянной (здесь для определенности мы выберем ее положительной).

Пусть $A^{I J}-S O(4,1)$-связность; здесь и далее индексы $I, J, \ldots$, обозначенные большими буквами, принимают значения $0,1,2,3,4$. Пусть $v^{I}$ - 0-форма, принимающая значения в пространстве $S O(4,1)$-векторов. Частица вводится в теорию аналогично $(2+1)$-мерной гравитации в $(1)$, но $h$ и $K$ теперь являются величинами из группы $S O(4,1)$, и вместо антидиагональной билинейной формы $\langle\cdot\rangle$ стоит обычный след.

Действие гравитации, взаимодействующей с частицей, записывается как

$$
S=\frac{l^{2}}{8 \pi G} \int \epsilon_{I J K L M} F^{I J}(A) v^{K} \wedge F^{L M}(A)+\lambda\left(v^{I} v_{I}-1\right)+\int_{\gamma} \operatorname{Tr}\left(h^{-1} d_{A} h K\right),
$$

где $F^{I J}(A)$ - 2-форма кривизны связности $A$. Второй член в $(2)$ представляет собой условие нормировки для $v^{I}$, которое вводится с помощью множителя Лагранжа. Последний член - действие частицы. В формуле (2) $l=1 / \sqrt{\Lambda}-$ космологическая длина.

Заменой обозначений первое слагаемое в (2) сводится к действию гравитации с космологической постоянной в форме Картана-Вейля плюс характеристика Эйлера. Введем связность $\omega^{I J}$, по отношению к которой $v^{I}$ ковариантно постоянна, $d_{\omega} v^{I}=0$. Ее кривизна $R^{I J}(\omega)$ есть $S O(3,1)$-кривизна в подгруппе стабильности $v^{I}$, так как $R^{I J}(\omega) v_{I}=0$. Затем введем тетраду

$$
e^{I}=l d_{A} v^{I}
$$

Это действительно тетрада, поскольку $e^{I} v_{I}=0$. Тогда $S O(4,1)$-связность может быть разложена как

$$
A^{I J}=\omega^{I J}-\frac{1}{l}\left(v^{I} e^{J}-v^{J} e^{I}\right)
$$

что ведет к следующему разложению кривизны:

$$
F^{I J}=R^{I J}+\frac{1}{l^{2}} e^{I} \wedge e^{J}+\frac{1}{l}\left(v^{J} d_{\omega} e^{I}-v^{I} d_{\omega} e^{J}\right) .
$$

Подставляя это выражение в (2), мы видим, что последнее слагаемое в (3), которое содержит кручение, не входит в действие. Первые два слагаемых дают действие гравитации, в частности, перекрестный член дает действие Картана-Вейля.

Варьируя действие по временно́й компоненте $A$, мы получаем следующий набор уравнений связи:

$$
d_{A}\left(\epsilon_{I J K L M} v^{K} F^{L M}\right)=\left(h K h^{-1}\right)_{I J} \delta^{3}\left(x-x_{\mathrm{p}}\right) .
$$


Используя эти уравнения, можно попробовать выразить энергию-импульс частицы, которые составляют трансляционную часть заряда в правой части (4), через некоторые интегральные характеристики геометрии.

Далее мы в ряде случаев будем использовать безындексные обозначения, например $(\epsilon v F)_{I J} \equiv \epsilon_{I J K L M} v^{K} F^{L M}$.

Мы можем взять произвольный шар $B$, содержащий нашу частицу, $x_{\mathrm{p}} \in B$, так, чтобы начало отсчета располагалось на его границе. Пусть $\gamma(x) \in S O(4,1)$ таково, что $\gamma\left(x_{\mathrm{p}}\right)=h$. Заряд $K$ может быть выражен через интеграл по шару:

$$
K=\int_{B} \gamma^{-1} d_{A}(\epsilon v F) \gamma .
$$

Чтобы преобразовать его в поверхностный интеграл, в общей ситуации нам потребовалось бы нечто вроде "неабелевой теоремы Гаусса", которой до сих пор нет. Однако, если $F$ - решение уравнений связей (4) с дельта-функцией в правой части, существует $\gamma$ такое, что

$$
K=\int_{B} \gamma^{-1} d_{A}(\epsilon v F) \gamma=\int_{\partial B} \gamma^{-1}(\epsilon v F) \gamma .
$$

Это получается просто потому, что дельта-функция есть обычная дивергенция некоторого векторного поля.

Последнее выражение напоминает формулу для магнитного заряда в теории Янга-Миллса [11], где $v$ играет роль поля Хиггса. Аналогия с решением 'т Хоофта-Полякова, однако, не полная. Во-первых, здесь магнитный заряд - матричная величина, так как калибровочная группа больше, чем $S O(3)$. Поэтому нам требуется калибровочное преобразование величины $\gamma(x)$ в (5), (6), чтобы поместить решение в подгруппу $S O(3)$ калибровочной группы. Второе отличие - это радиальная зависимость поля Хиггса. Тогда как в решении 'т Хоофта-Полякова поле Хиггса стремится к константе на бесконечности, здесь $v^{I}$ имеет смысл координатной сетки в пространстве де Ситтера. Если мы выберем статическую систему координат, поле примет следующую форму:

$$
l v=\left(\sqrt{l^{2}-r^{2}} \operatorname{sh} \frac{t}{l}, r n^{i}, \sqrt{l^{2}-r^{2}} \operatorname{ch} \frac{t}{l}\right),
$$

где $i=1,2,3$ и $n^{i}=x^{i} /|r|$ - так называемый "еж". В системе отсчета, где частица покоится, решение можно искать в виде

$$
A^{i j}=f(r)\left(n^{i} d n^{j}-n^{j} d n^{i}\right), \quad A^{i 0}=\operatorname{sh} \frac{t}{l} g(r) d n^{i}, \quad A^{i 4}=\operatorname{ch} \frac{t}{l} g(r) d n^{i} .
$$

Здесь решение ищется в калибровке $A_{r}=0$. В (8) $f(r)$ и $g(r)$ - неизвестные функции от $r$, которые надо найти. Однако эти функции не появляются в окончательном результате. В силу известной формулы

$$
\epsilon_{i j k} d n^{i} \wedge d n^{j} \wedge d n^{k}=4 \pi \delta^{3}(x),
$$

в конце мы должны получить степень Браувера в (6):

$$
K=\int_{\partial B} \gamma^{-1}\left(\epsilon_{I J k l m} n^{k} d n^{l} \wedge d n^{m}\right) \gamma,
$$


где значение $\gamma$ может быть выбрано постоянным на $\partial B$. Чтобы компенсировать в (7) возрастание "поля Хиггса", как $r$, кривизна должна убывать, как $1 / r^{3}$. Известно, что это действительно так для решения Шварцшильда.

Теперь мы можем использовать факт, что формула (9) инвариантна относительно произвольных непрерывных преобразований поля $n^{i}$, сохраняющих его норму. Выражение (10) можно существенно упростить, если преобразовать $n^{i}$ в постоянное поле на сфере ("причесать ежа") и вынести его за знак интеграла, а к тому, что останется под интегралом, применить теорему Стокса. Будь это возможно, результат в (10) был бы нулевым, так как сфера не имеет границы. Однако преобразование из "ежа" в постоянное поле $n^{i}$ не может быть сделано непрерывным на всей сфеpe, поскольку оно изменяет число намоток с 1 до 0. Но это преобразование может быть сделано непрерывным на любом открытом подмножестве сферы. Так, мы можем разрезать сферу пополам и "причесать ежа" на каждой из половинок [12] или, как альтернатива, сделать в сфере прокол и "причесать ежа" на проколотой сфере [13]. Направление постоянного поля $n^{i}$, совпадает с направлением от частицы до начала отсчета. Теперь мы можем применить теорему Стокса к кривизне в подынтегральном выражении в (6) (теперь $v^{I}$ - константа). Область интегрирования имеет границу и топологически нетривиальную петлю вдоль нее: экватор в первом случае из указанных выше и прокол во втором. Оба случая дают одинаковый результат для петли Вильсона:

$$
g=h \exp \left[\epsilon_{a b c 0} 2 G m \frac{x^{c}}{x^{2}}\right] h^{-1} .
$$

В пределе $l \rightarrow \infty$ мы имеем $x^{a}=\left(t, x^{i}\right), g$ - элемент подгруппы $S O(3,1)$ и $K=l m T^{04}$.

Кривизна импульсного пространства меняется с расстоянием до начала отсчета. Для заданного $r=\sqrt{x^{2}}$ радиус кривизны импульсного пространства есть такая энергия, для которой $r$ - радиус Шварцшильда. Петля (11) принадлежит классу сопряженности чисто вращательного элемента подгруппы Картана. Это означает, что одно бустово направление отсутствует, имеются только два некомпактных направления в импульсном пространстве.

\section{4. ЗАКЛЮЧЕНИЕ}

Можно ожидать, что кривизна импульсного пространства улучшит ультрафиолетовое поведение квантовой теории гравитации и даже сделает ее перенормируемой. Этот эффект является непертурбативным, и он потребовал бы точного решения теории. Для четырехмерной гравитации это вряд ли возможно. Альтернативная возможность - модифицировать теорию возмущений так, чтобы учитывалась кривизна импульсного пространства. Такой подход для трехмерной гравитации, взаимодействующей со скалярным полем, был применен в работе [14]. Было бы интересно распространить эти результаты на $(3+1)$-мерную гравитацию.

Благодарности. Автор благодарит организаторов конференции "В поисках фундаментальных симметрий”, посвященной 90-летию со дня рождения Ю. В. Новожилова, а также выражает свою благодарность А. Андрианову, А. Головневу и С. Пастону за обсуждения. 


\section{Список литературы}

[1] J. Ambjørn, J. Jurkiewicz, R. Loll, Phys. Rev. Lett., 85:5 (2000), 924-927, arXiv: hep-th/0002050; 93:13 (2004), 131301, 4 pp., arXiv: hep-th/0404156; Phys. Rev. D, 72:6 (2005), 064014, 24 pp., arXiv: hep-th/0505154.

[2] S. Weinberg, "Ultraviolet divergences in quantum theories of gravitation", General Relativity: An Einstein Centenary Survey, eds. S. W. Hawking, W. Israel, Cambridge Univ. Press, Cambridge, 1979, 790-831; M. Reuter, F. Saueressig, Phys. Rev. D, 65:6 (2002), 065016, 26 pp., arXiv: hep-th/0110054; D. F. Litim, Phys. Rev. Lett., 92:20 (2004), 201301, 4 pp., arXiv: hep-th/0312114; M. Niedermaier, Class. Quantum Grav., 24:18 (2007), R171-R230, arXiv: gr-qc/0610018.

[3] S. Carlip, AIP Conf. Proc., 1196:1 (2009), 72-80, arXiv: 0909.3329.

[4] М. П. Бронштейн, "Квантовая теория слабых гравитационных полей", Эйнштейновский сборник (1980-1981), ред. И. Ю. Кобзарев, Наука, М., 1985, 267-282.

[5] G. 't Hooft, Class. Quantum Grav., 10:8 (1993), 1653-1664; 13:5 (1996), 1023-1039.

[6] H. J. Matschull, M. Welling, Class. Quantum Grav., 15:10 (1998), 2981-3030, arXiv: gr-qc/9708054.

[7] И. Я. Арефьева, ТМФ, 43:1 (1980), 111-116.

[8] A. Yu. Alekseev, A.Z. Malkin, Commun. Math. Phys., 169:1 (1995), 99-119, arXiv: hep-th/9312004; C. Meusburger, B. J. Schroers, Nucl. Phys. B, 738:3 (2006), 425-456, arXiv: hep-th/0505143.

[9] G. Amelino-Camelia, D. V. Ahluwalia, Internat. J. Modern Phys. D, 11:01 (2002), 35-59, arXiv: gr-qc/0012051; J. Kowalski-Glikman, "Introduction to doubly special relativity", Planck Scale Effects in Astrophysics and Cosmology, Lecture Notes in Physics, 669, eds. J. Kowalski-Glikman, G. Amelino-Camelia, Springer, Berlin, 2005, 131-159, arXiv: hep-th/0405273.

[10] S. W. MacDowell, F. Mansouri, Phys. Rev. Lett., 38:14 (1977), 739-742; Erratum, 38:23 (1977), 1376.

[11] G. 't Hooft, Nucl. Phys. B, 79:2 (1974), 276-284; А. М. Поляков, Писъма в ЖКЭТФ, 20:6 (1974), 430-433.

[12] T. T. Wu, C. N. Yang, Phys. Rev. D, 12:12 (1975), 3845-3857.

[13] P. A. M. Dirac, Proc. Roy. Soc. A, 133:821 (1931), 60-72.

[14] L. Freidel, E. R. Livine, Phys. Rev. Lett., 96:22 (2006), 221301, 4 pp., arXiv: hep-th/ 0512113. 